\title{
System Design for Automated Time Study of Nursing
}

\author{
Shohei Nakamura, Ichiroh Kanaya, Yuko Ohno, and Kazuo Kawasaki \\ Osaka Universiy \\ 2-1 Yamadaoka Suita, Osaka, Japan
}

\begin{abstract}
There has been a shortfall in the number of nurses, so it is required to optimize the nursing service. Then, the authors proposed a new system design for automated time study of nursing to investigate nursing service easily. The system combines images from video monitoring and information from ambient sensing, and it analyzes them after combining. Besides the authors conducted video monitoring with ambient sensing in a hospital and analyzed images from the video monitoring. The authors indicated the effectiveness and potential of the system.
\end{abstract}

Keywords: Time study, Nursing, Video monitoring, Image analysis, Ambient sensing.

\section{Introduction}

Recently, there has been a shortfall in the number of nurses in Japan. Thus, in order to treat patients adequately with limited human resources, optimizing nursing service is required in medical site. To optimize nursing service, at first, to figure out precisely the present situation of nurses is needed; this must be based on a quantitative and detailed analysis.

Time study is just a method to record the content of work in detail and to provide data for analysis of the current circumstances. On a traditional time study, one observer always follows one subject on her or his job and documents it in detail. Normally the observed object of time study is a team of about $10 \sim 20$ nurses, so at least 20 members are required for conducting time study, considering backup members. Therefore, this method needs large amount of well-trained human resources and burdens nurses both physically and mentally because of following them constantly. For these reasons, the new method of time study which clear these problems is strongly demanded. In this research, the authors proposed new method of time study and examined it in clinical practice.

\section{Method}

The new method of time study is required to get enough information for analysis with minimal equipments after solving the traditional method's problems. The information needed in time study is $5 \mathrm{~W}$ information (What, When, Where, Who, Whom) about nurses' job. In this research, the authors proposed the system for time study that using 
Video monitoring, Ambient sensing and Image analysis organically. Video monitoring is to record nursing service by some cameras located adequate place. It can get $5 \mathrm{~W}$ information at the same time but it has some restrictions about installation location, angle of view or background. To complement it, the authors use small sensors or devices. Ambient sensing is one method to support video monitoring and to get information that can't be got from only video monitoring by setting up them on desks, walls or nurses. After taking these data, they are integrated, processed and analyzed in computer. This system mainly uses differential analysis, because the moving object can be extracted easily. It can accomplish the automated time study with solving the traditional time study's problem if all attempts success.

\section{Results and Discussion}

For demonstrating the efficiency of this system, Video monitoring and Ambient sensing was conducted at Saito Yukokai hospital. The purpose of this time study was to investigate the work of managing the narcotic drug and anti cancer drug, because these works that should be controlled strictly were one reason to tie up the nurses. Therefore, the author set eight video cameras centering around the coffer of the drugs at the nurse station with careful attention to installation place not to burden nurses. Besides, the night nurse in this nurse station used a red LED device and a welsupport. In this research, LED device was used as an optical marker for picking out the object nurse from background in differential analysis. The spec of the red LED was that the luminance was $3000 \mathrm{mcd}$, the angle of light direction was $120 \mathrm{deg}$ and covered by tracing paper to diffuse its light. Welsupport is high functional pedometer on shelves by NIPRO. It can measure movement of nurse, so it was used for measuring movement performed outside angle of view and total amount of nurse's movement.

Below figure shows the representative development of differential analysis. This difference images were calculated with 1 / 29.27 second-differential images. It could extract a body movement of nurse clearly. Moreover, it could extract specifically a light of LED device. A red strap in image before process was changed color into green in image after process, but the color of red LED was not changed. From results of graph of welsupport, its data roughly synchronized with actual movement of nurse, but it could use only for assistant information.
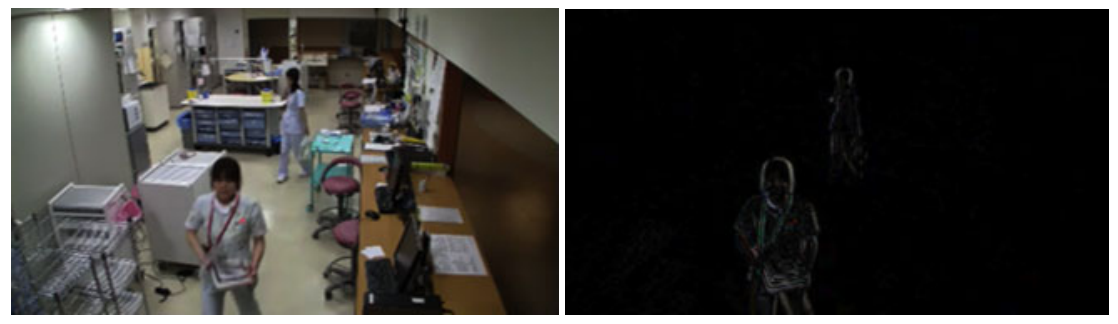

Fig. 1. Images before and after in differential analysis 